\title{
Pediatrics
}

\section{Developmental coordination disorder and the COVID-19 pandemic: Some considerations}

\author{
Jorge Lopes Cavalcante Neto ${ }^{1}$ (i) , Tatiane Targino Gomes Draghi ${ }^{2}$ \\ ${ }^{1}$ Universidade do Estado da Bahia, Departamento de Ciências Humanas, Jacobina, BA, Brasil; \\ ${ }^{2}$ Universidade Federal de São Carlos, Departamento de Fisioterapia, São Carlos, SP, Brasil. \\ Associate Editor: Angelina Zanesco. UNESP/Rio Claro, SP, Brasil.
}

\begin{abstract}
Developmental Coordination Disorder (DCD) is a developmental disorder characterized by significant poor motor performance, interfering in children's health, participation, and quality of life at home, school, or playground contexts. According to the literature, children with DCD are at greater risk of anxiety and depression symptoms compared to their typically developing peers. Due to the COVID-19 pandemic, many children with DCD are isolated at home and diminishing their opportunity to participate in different types of activities. In addition, this scenario can increase the symptoms of anxiety and depression in these children, which warns us to state some important considerations in this brief communication. As well, some important recommendations about activities to perform at home with relatives, such as active video games or ball games, were made to help these children and their parents in this complex and challenging scenario worldwide. Therefore, this paper summarizes some considerations regarding DCD and the COVID-19 pandemic.
\end{abstract}

Keywords: developmental coordination disorder; motor development; children

\section{Introduction}

In March 2020, the World Health Organization (WHO) began to regard the COVID-19 outbreak as a pandemic ${ }^{1}$. Since then, cities around the world have implemented more or less restrictive social isolation policies to decrease contamination among citizens and prevent a breakdown of healthcare systems and services. According to the Centers for Disease Control and Prevention ${ }^{2}$, groups at higher risk for infection and illness related to COVID-19 include older adults and individuals of any age with underlying medical conditions, such as asthma, chronic kidney disease, chronic lung disease, diabetes, hemoglobin disorders, liver disease, serious heart conditions, severe obesity, or suppressed or compromised immune systems.

A recent systematic review of forty-five scientific papers ${ }^{3}$ found that children with COVID-19 present symptoms comparable to those observed in adults (e.g., fever, respiratory complications) and respond similarly to treatment. Furthermore, children displayed lower inflammatory markers, indicating a less severe effect on their overall health and a more positive prognosis. However, we do not yet have the research needed to understand the potential effects of COVID-19 infection in children. Knowledge about COVID-19, including effective treatments and long-term consequences, is still scarce. Thus, while the initial impact of the virus may be milder in children, it could interfere with important aspects of child development.

Children with developmental disorders, such as Developmental Coordination Disorder (DCD), are particularly vulnerable to long-term complications related to COVID-19. DCD is one of the most complex and prevalent childhood motor skills disorders, affecting around $6 \%$ of school-aged children ${ }^{4}$. The condition is characterized by significantly lower motor performance not related to an intellectual or visual disability or known neurological condition that interferes with activities of daily living as well as academic and recreational endeavors ${ }^{4,5}$. The lack of motor performance associated with DCD widely restricts participation, thereby negatively affecting the quality of life from childhood into adulthood. Individuals with DCD frequently self-identify as less skilled than their peers and thus avoid collective athletic activities (e.g., sports) in favor of sedentary ones (e.g., watching television) ${ }^{6}$.

These negative self-perceptions may be further reinforced by judgments and comparisons from typically developing peers and family members, creating a vicious cycle ${ }^{6}$, in which consequences in their mental health are expected. A recent systematic review synthesizing results from six original studies found significantly greater symptoms of anxiety and depression in children with DCD compared to typically developing peers ${ }^{7}$. The sedentary lifestyle typical to children with DCD can also lead to obesity with a high risk of metabolic disease or cardiac complications ${ }^{6}$. Children with DCD have thus been shown to have reduced opportunities to improve motor performance, increase levels of physical activity, and initiate or maintain friendships ${ }^{8}$. As explained by the environmental stress hypothesis ${ }^{8}$, this cascade of biopsychosocial consequences is the secondary stressor from the motor deficit, which is the primary stressor in children with DCD. 
In the context of the COVID-19 pandemic, social isolation has forced families around the world to shelter in place, with the duration of the pandemic remaining unclear. Feelings of loneliness and decreased mental wellbeing, such as poor self-esteem and symptoms of anxiety and depression, are accordingly expected ${ }^{9}$. Individuals are also more likely to increase the amount of time they spend engaging in sedentary activities, which could lead to secondary health consequences ${ }^{6}$. Those who are already at risk for poor physical and mental health are even more vulnerable?. The combination of impaired motor performance and restrictive environmental factors could lead to physical, psychological, and psychosocial issues for children with DCD both during and after the pandemic. It is therefore essential that children with DCD be motivated to engage in physical activities. If they do not have such opportunities, their motor functions cannot improve.

It is not yet known if or when socialization will resume as before. When will people again be "free"? When will children return to schools and collective recreational activities? Many unanswered questions remain, and, in the meantime, the overall health and quality of life of children with DCD could be negatively impacted. How has the pandemic affected their mental health? Do they have adequate opportunities to participate in physical activities?

\section{Some Recommendations}

Engaging children with DCD in a health regimen can promote increased participation in physical activities, stimulate them to be more physically active, improve their self-confidence, and mitigate or eliminate symptoms related to mental health problems. When used as recommended, active video games can be an effective option for children with DCD who experience motor and/or functional challenges.

Active video games provide immediate feedback, allowing players to process frustration regarding their lack of skills and abilities as they progress. They also give players rewards for achievement, encouraging self-confidence. Studies also support the promising effects of active video games in improving motor performance ${ }^{10}$, energy expenditure ${ }^{11}$, and mental health outcomes ${ }^{6}$ in children. Further, most active video games are commercially available, low cost, secure, and can be used at home, even in small spaces. We, therefore, advocate for the use of active video game systems and devices, such as the Nintendo Wii, Microsoft Xbox Kinect, Sony PlayStation 3 Move, and Sony PlayStation 2 EyeToy, as a novel but effective means of preventing negative physical and mental health outcomes related to COVID-19 in children with DCD.

Another accessible option is to provide specific motor tasks. These could include playing with balls, bowling using pins made from bottles, and walking or running routes determined by wooden rods or adhesive tape along the floor. All these tasks can be performed by children with DCD at home. The motor skills required to perform them can improve their general motor performance and balance, as attested in the literature ${ }^{10}$. Parents or guardians can also engage in these activities with their children as well as activities designed to stimulate self-care or domestic wellbeing, such as gardening, cooking, etc. These activities, in addition to increasing motor skills and self-confidence, can strengthen the family bond by providing time for family socialization, which may have been lacking previously due to various life commitments. Such moments can also yield positive mental health outcomes, effectively relieving the symptoms of anxiety and depression commonly observed in children with DCD. In this way, these children may experience fewer negative consequences as a result of the COVID-19 pandemic.

\section{References}

1. Dong E, Du H, Gardner L. An interactive web-based dashboard to track COVID-19 in real-time. Lancet, Infect. dis. 2020; 20(5):533-4. doi:10.1016/S1473-3099(20)30120-1.

2. Centers for Disease Control and Prevention. People Who Are at Higher Risk for Severe Illness, Coronavirus, COVID-19. Available from:https://www.cdc.gov/coronavirus/2019-ncov/ need-extra-precautions/groups-at-higher-risk.html[Accessed4thJune 2020].

3. Ludvigsson JF. A systematic review of COVID-19 in children shows milder cases and a better prognosis than adults. Acta Paediatr. 2020; 109(6):1088-95. doi:10.1111/apa.15270.

4. Blank R, Barnett AL, Cairney J, Green D, Kirby A, Polatajko H, et al. International clinical practice recommendations on the definition, diagnosis, assessment, intervention, and psychosocial aspects of developmental coordination disorder.Dev. Med. Child. Neurol.2019; 61(3):242-85. doi:10.1111/dmcn.14132.

5. American Psychiatric Association. Diagnostic and Statistical Manual of Mental Disorders. Washington, DC, Fifth Edition, 2013.

6. Straker LM, Campbell AC, Jensen LM, Metcalf DR, Smith AJ, Abbott RA, et al.Rationale, design and methods for a randomised and controlled trial of the impact of virtual reality games on motor competence, physical activity, and mental health in children with developmental coordination disorder. BMC Public Health. 2011; 11(1):654. doi: 10.1186/1471-2458-11-654.

7. Draghi TTG, Cavalcante Neto JL, Rohr LA, Jelsma LD, Tudella E. Symptoms of anxiety and depression in children with developmental coordination disorder: a systematic review. J. Pediatr.2019; 96(1): 8-19. doi:10.1016/j.jped.2019.03.002.

8. Cairney J, Rigoli D, Piek J. Developmental coordination disorder and internalizing problems in children: The environmental stress hypothesis elaborated. Dev. Rev.2013; 33(3):224-38. doi:10.1016/J.DR.2013.07.002.

9. Banerjee D, Rai M. Social isolation in Covid-19: The impact of loneliness.Int. J. Soc.Psychiatry.2020; 66(6): 525-7. doi:10.1177/0020764020922269.

10. Cavalcante Neto JL, de Oliveira CC, Greco AL, Zamunér AR, Moreira RC, Tudella E. Is virtual reality effective in improving the motor performance of children with developmental coordination disorder? A systematic review.Eur. J. Phys. Rehabil. Med.2019; 55(2):291-300. doi:10.23736/ S1973-9087.18.05427-8.

11. Foley L, Maddison R. Use of active video games to increase physical activity in children: a (virtual) reality? Pediatr. Exerc. Sci. 201022(1):7-20. doi:10.1123/pes.22.1.7. 
12. Cavalcante Neto JL, Steenbergen B, Wilson P, Zamunér AR, Tudella E. Is Wii-based motor training better than task-specific matched training for children with developmental coordination disorder? A randomized controlled trial.Disabil. Rehabil. 2019; 42(18):2611-20.doi:10.1080/09638288.2019.1572794.

\section{Corresponding author}

Jorge Lopes Cavalcante Neto

State University of Bahia, J.J Seabra street, 158, Estação. 44700-000. Jacobina, Bahia, Brasil. Telephone: (+55) (74) 3621-3337

Email: jorgelcneto@hotmail.com
Manuscript received on December 12, 2020

Manuscript accepted on January 4, 2021

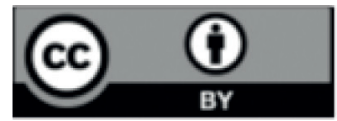

Motriz. The Journal of Physical Education. UNESP. Rio Claro, SP, Brazil - eISSN: 1980-6574 - under a license Creative Commons - Version 4.0 